\title{
Dependently-Typed Data Plane Programming
}

\author{
MATTHIAS EICHHOLZ, Technical University of Darmstadt, Germany \\ ERIC HAYDEN CAMPBELL, Cornell University, USA \\ MATTHIAS KREBS, Technical University of Darmstadt, Germany \\ NATE FOSTER, Cornell University, USA \\ MIRA MEZINI, Technical University of Darmstadt, Germany
}

Programming languages like P4 enable specifying the behavior of network data planes in software. However, with increasingly powerful and complex applications running in the network, the risk of faults also increases. Hence, there is growing recognition of the need for methods and tools to statically verify the correctness of P4 code, especially as the language lacks basic safety guarantees. Type systems are a lightweight and compositional way to establish program properties, but there is a significant gap between the kinds of properties that can be proved using simple type systems (e.g., SafeP4) and those that can be obtained using full-blown verification tools (e.g., p4v). In this paper, we close this gap by developing П4, a dependently-typed version of P4 based on decidable refinements. We motivate the design of $\Pi 4$, prove the soundness of its type system, develop an SMT-based implementation, and present case studies that illustrate its applicability to a variety of data plane programs.

CCS Concepts: • Software and its engineering $\rightarrow$ Formal language definitions; $\bullet$ Networks $\rightarrow$ Programming interfaces.

Additional Key Words and Phrases: Software-Defined Networking, P4, Dependent Types

\section{ACM Reference Format:}

Matthias Eichholz, Eric Hayden Campbell, Matthias Krebs, Nate Foster, and Mira Mezini. 2022. DependentlyTyped Data Plane Programming. Proc. ACM Program. Lang. 6, POPL, Article 40 (January 2022), 28 pages. https://doi.org/10.1145/3498701

\section{INTRODUCTION}

Computer networks are becoming increasingly programmable as languages like P4 [Bosshart et al. 2014] make it possible to specify the behavior of data planes in software. With the increased availability of programmable devices, a number of powerful and complex applications having become viable, ranging from novel network protocols to full-blown in-network computation (e.g., executing application-level storage queries using network devices [Jin et al. 2017]). But as the complexity of these applications increases, so does the risk of faults, especially as P4's main abstraction for representing packet data-namely header types-lacks basic safety guarantees. Experience with a growing number of programs has shown the risks of the unsafe approach, which often leads to subtle software bugs [Eichholz et al. 2019; Liu et al. 2018]. This is clearly unacceptable,

Authors' addresses: Matthias Eichholz, Technical University of Darmstadt, Germany, eichholz@cs.tu-darmstadt.de; Eric Hayden Campbell, Cornell University, USA, ehc86@cornell.edu; Matthias Krebs, Technical University of Darmstadt, Germany, krebs@cs.tu-darmstadt.de; Nate Foster, Cornell University, USA, jnfoster@cs.cornell.edu; Mira Mezini, Technical University of Darmstadt, Germany, mezini@cs.tu-darmstadt.de.

This work is licensed under a Creative Commons Attribution 4.0 International License.

(c) 2022 Copyright held by the owner/author(s).

2475-1421/2022/1-ART40

https://doi.org/10.1145/3498701

Proc. ACM Program. Lang., Vol. 6, No. POPL, Article 40. Publication date: January 2022. 
given the crucial role that networks play in nearly all modern systems. Hence, we need methods and tools to statically verify the correctness of data plane programs.

Today, most data plane verification tools [Dumitrescu et al. 2020; Liu et al. 2018; Stoenescu et al. 2018] are monolithic in nature. For example, p4v [Liu et al. 2018], which is based on software model checking, operates on whole programs. But while monolithic approaches have certain advantagese.g., they minimize the need for programmer annotations-they also have downsides. The most fundamental limitation is the inherent tension with modular design-it is difficult to accommodate an "open-world" model, in which third-party components are plugged into existing programs. For instance, an equipment vendor might want to provide a "base" program that implements standard packet-processing functionality like Ethernet switching, and allow customers to add custom functionality of their own design [Baldi 2019; Gao et al. 2020; Soni et al. 2020]. Composable approaches to data plane programming require compositional reasoning methods [Beckett and Mahajan 2020].

Type systems are a lightweight and compositional way to establish program properties-i.e., the types for individual components document assumptions about the components they rely upon as well as the guarantees they offer. However, somewhat surprisingly, types have rarely been applied in the realm of network programming, and the few exceptions [Eichholz et al. 2019; Ennals et al. 2004; Muthukrishnan et al. 2010] are simple type systems with limited expressive power. For example, SafeP4 [Eichholz et al. 2019] uses regular types [Castagna et al. 2014; Gapeyev and Pierce 2003; Hosoya and Pierce 2003] and path-sensitive occurrence typing [Tobin-Hochstadt and Felleisen 2010] to reason about basic safety properties, but it cannot capture richer program properties (e.g., whether the IPv4 and IPv6 headers are only ever accessed on mutually exclusive execution paths), or track the values of individual fields (e.g., whether EtherType equals to $0 \times 0800$, which indicates an IPv4 packet, or to $0 \times 86 \mathrm{DD}$, which indicates an IPv6 packet). The inability of SafeP4 to reason about the values being manipulated by the program significantly limits its expressiveness. In general, there is a significant gap between the kinds of properties that can be checked using type systems like SafeP4 and full-blown verification tools like $\mathrm{p} 4 \mathrm{v}$.

Thus, it is natural to ask whether we can design a compositional type system that has the same expressive power as data plane verification tools. This paper answers this question in the affirmative, by presenting П4-a dependently-typed version of $\mathrm{P} 4$. П4 fits with the trend of recently proposed dependently-typed languages [Condit et al. 2007; Rondon et al. 2008; Vazou et al. 2014; $\mathrm{Xi}$ and Pfenning 1999] that are blurring the line between type checking and theorem proving. For instance, Liquid Haskell [Rondon et al. 2008; Vazou et al. 2014] allows programmers to smoothly shift from properties that can be checked with traditional typing disciplines to more sophisticated ones that go beyond simple syntactic checks. Under the hood, Liquid Haskell uses an SMT solver to automatically discharge the logical proof obligations generated during type checking.

Yet, thus far, the dependently-typed approach has not been explored for network programming. In this paper, we demonstrate that data plane programs are a "killer application" for dependent types. On the one hand, they clearly need precise types, as most programs rely on intricate packet formats (e.g., so-called "type-length-value" encodings, where the first few bits determine the type, length, and structure of the bits that follow). On the other hand, data plane programs are fundamentally simple (e.g., P4 does not support pointers or loops) and lack the kinds of complex features that often make precise type systems complicated to design and implement.

Our main contribution lies in exploring and addressing the subtle challenges that arise in developing a dependent type system for the $\mathrm{P} 4$ programming language, including balancing the tradeoffs between expressiveness and decidability. $\Pi 4$ features a combination of refinement types, dependent function types, union types, and explicit substitutions. This combination is key to retain precision during type checking-e.g., we can compute exact types for conditionals, thereby having 
access to an accurate type at any program point. Moreover, our design enables precise typing in the presence of domain-specific features that combine packet serialization and deserialization operations with imperative control-flow. To this end, $\Pi 4$ combines a dependent sum type with a novel "chomp" operation that computes the type that remains after extracting bits from a packet buffer. We formally prove that $\Pi 4$ 's type system is sound via standard progress and preservation theorems.

The chomp operator is reminiscent of regular expression derivatives [Brzozowski 1964]. To the best of our knowledge, with a few notable exceptions (e.g., work by McBride [McBride 2001]) derivative-like operations have not been extensively studied the context of dependent type systems. Thus, beyond providing an elegant solution to a practical problem, we believe that $\Pi 4$ 's innovative combination of dependent types with regular types and the possibility to compute derivatives of types is of general theoretical interest and may be useful in other domains-e.g., one potential direction is verified serializers and deserializers [Delaware et al. 2019; Ramananandro et al. 2019].

We have built a prototype implementation of $\Pi 4$ in OCaml and the Z3 SMT solver. The type checker determines whether a $\Pi 4$ program has a given type by checking the validity of a series of logical formulae using an SMT solver. We encode types into the effectively propositional fragment of first-order logic over fixed-width bit vectors, which facilitates automating subtyping and equivalence checks. We prove (cf. Theorem 4.1) that this logical fragment is sufficient for checking our types under the assumption that the types written by the programmer denote finite sets. We believe this is a reasonable assumption, because networks enforce a maximum transmission unit (MTU) (i.e., a bound on the size of packets constraining the maximum number of bits that network switches can receive or transmit ${ }^{1}$ ) which bounds the size of bit vectors we need to consider in the encoding. In the presence of an MTU, our types denote finite sets, which can be enumerated to decide the key judgments (i.e., subtyping, size constraints, and inclusion checks).

Using our $\Pi 4$ prototype, we develop several case studies, demonstrating that $\Pi 4$ is capable of expressing and (modularly) reasoning about properties from the literature ranging from basic safety to intricate invariants: parser round-tripping, protocol conformance, determined forwarding, etc. We selected properties that are also studied by recent data plane verification tools like $\mathrm{p} 4 \mathrm{v}$ or Vera, which indicates that $\Pi 4$ is capable of covering properties of interest to the networking community. However, we leave a careful study of the practical utility of $\Pi 4$ (e.g., with larger examples and user studies) to future work.

Overall, the contributions of our work are as follows:

- Section 2 motivates dependently-typed data plane programming.

- Section 3 presents $\Pi 4$, a dependently-typed core of P4, featuring a combination expressive types for describing structures (regular types as well as decidable refinement and dependent function types) combined with a bit-by-bit "chomp" operator.

- Section 3 develops a semantic proof of soundness for П4's type system.

- Section 4 defines a decidable algorithmic type system for $\Pi 4$.

- Section 5 and Section 6 discusses case studies using П4's type system to check realistic program properties.

\section{BACKGROUND}

Most networks are based on a division of labor between two components: the control plane and the data plane. The control plane, usually implemented in software, is responsible for performing tasks such as learning the topology, computing network-wide forwarding paths, managing shared

\footnotetext{
${ }^{1}$ The MTU is often set to 1500 bytes.
} 


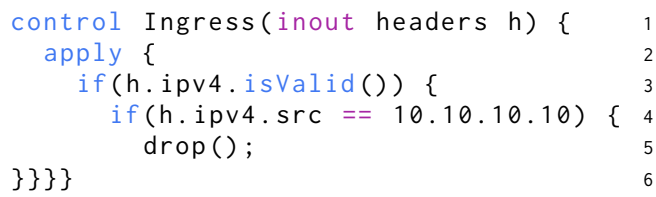

(a) Explicit validity check

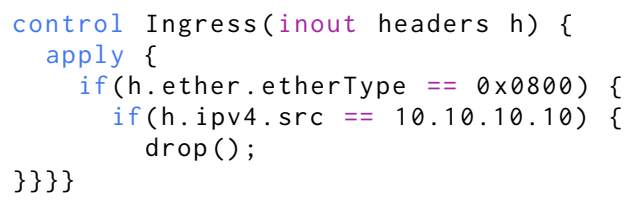

(b) Implicit validity check

Fig. 2. Safe implementation of ingress

In practice, however, relying on explicit validity checks is not sufficient. For example, consider the code shown in Figure 2b. Recall that, given the parser above, the IPv4 header will be present if the EtherType is $0 \times 0800$. Hence, the ingress control can be safely executed. Yet, SafeP4's type checker rejects the program because the type system is not expressive enough to capture the dependency between the EtherType value and IPv4's validity.

To address this problem, П4 employs a dependent type system [Xi and Pfenning 1999], in which we can compute a precise type for the program after parsing:

$$
\left(x:\left\{y: \epsilon|| y \cdot \operatorname{pkt}_{\text {in }} \mid>272\right\}\right) \rightarrow\left(\begin{array}{l}
\Sigma y: \text { ether. }\{z: \text { ipv } 4 \mid y \cdot \text { ether.etherType }==0 x 0800\} \\
+\{z: \text { ether } \mid z \cdot \text { ether.etherType } \neq 0 x 0800\})
\end{array}\right)
$$

Intuitively, this type says that, starting with the empty heap $(y: \epsilon)$ and a packet buffer that has at least enough bits to extract both the Ethernet and the IPv4 header $\left(\left|y \cdot p k t_{i n}\right|>272\right)$, the parser ends in one of two possible states: (1) either both Ethernet and IPv4 are valid $(\Sigma y$ : ether. $\{z:$ ipv $4 \mid \ldots\})$, if the EtherType is equal to $0 \times 0800$ (note how $z$ : ipv4 is conditioned by $y$.ether.etherType $==0 \times 0800$ ), or (2) just Ethernet is valid, if EtherType is not equal to $0 \times 0800$. When checking the ingress control, the type checker uses the predicate ether.etherType $==0 \times 0800$ from the conditional to derive the set of valid header instances, which, in this case, includes IPv4. Thus, accessing the IPv4 source address is safe and the program correctly passes the type checker.

While the output type is admittedly notationally heavy-a common feature in precise type systems-note that the programmer is not forced to write down the most precise type! П4 only requires the annotated type to be sufficiently precise to capture basic safety guarantees and other desired invariants. For example, in a program where only the Ethernet header needs to be valid at the end of the parser, they could use the type $\left(x:\left\{y: \epsilon|| y \cdot p k t_{\text {in }} \mid>272\right\}\right) \rightarrow$ ether , which indicates that, at the end of the parser, at least Ethernet is valid (and possibly others, too).

This example illustrates how $\Pi 4$ 's type system statically checks intricate safety properties with high precision. Sections 5 and 6 present more case studies showing how $\Pi 4$ 's type system can be used to check practical properties of interest.

\section{DEPENDENT TYPES FOR P4 (П4)}

This section introduces the design, syntax, and semantics of $\Pi 4$, a core calculus modeled after $\mathrm{P} 4$ and equipped with dependent types.

\subsection{Design of $\Pi 4$}

$\Pi 4$ focuses on the aspects of the P4 programming language that benefit from dependent types, (e.g., parsing, deparsing, validity, and control flow) and omits features that add clutter (e.g., externs, registers, checksums, hashing, and packages). Following p4v [Liu et al. 2018], we do not explicitly model match-action tables and instead use ghost state and conditionals to encode them (see 
Section 6). Consequently, $\Pi 4$ is a loop-free ${ }^{2}$ imperative language with a few domain-specific primitive commands: extract $(\iota)$, remit $(\iota)$, add $(\iota)$, and reset.

In $\mathrm{P} 4$, the emit $(l)$ primitive serializes a header instance $\iota$ into a bitstring and prepends it to the outgoing packet payload, only if $\iota$ is valid, otherwise it does nothing. To simplify typing rules and semantics, $\Pi 4$ provides the primitive remit $(\iota)$, which really emits $\iota$ if it is valid, and otherwise gets stuck. Hence, emit $(\iota)$ can be encoded as syntactic sugar: if ( $\iota$.valid) then remit $(\iota)$ else skip. Another superficial difference is that we model header field accesses as direct bit-slices into the instance (to avoid another layer of indirection in our semantics)-i.e., eth. srcAddr is written eth [48:96].

More substantially, П4 diverges from $\mathrm{P} 4$ in the way it handles instance validity. A header instance is valid in two cases: (i) if it has been extracted from the packet (which automatically populates the instance with the appropriate bits) or (ii) if its validity bit has manually been set using the setValid() method (which does nothing to the instance itself). In P4, reads to uninitialized variables produce undefined values, so a common programming practice is to follow a call to setValid() with a sequence of assignments to the header fields-thereby avoiding undefined reads. In $\Pi 4$, rather than forcing the programmer to manually write default values, the add $(\iota)$ command sets $\iota$.valid to true, and sets $\iota$ a pre-determined default value (say 0 ). ${ }^{3}$ If required, $\mathrm{P} 4$ 's behavior could be encoded using an extra 1-bit header to independently track the validity of the instance and initialization of its fields.

We also introduce a new primitive, reset, which models the behavior of $\mathrm{P} 4$ between pipeline stages. In many switch architectures [Bosshart et al. 2013], packets are deparsed and then reparsed between pipelines-e.g., after ingress and before egress. The reset command encodes the behavior of the inner step: it combines the deparsed bits with the packet's unparsed payload and passes it along as the input to the next stage. The reset command would also be useful to reason about invariants across multiple switch programs, although we don't explore that use in this paper.

Finally, in designing $\Pi 4$, our primary goal is to enable data plane programmers to make use of dependent types to verify useful program properties in a compositional way and without having to write manual proofs. To enable modular reasoning, we need a way to annotate (and modularly check) programs with types. We annotate a program $c$ with a type $\sigma$ using an ascription operator: $c$ as $\sigma$. The ascription has no effect on the runtime behavior of the code (i.e., $c$ as $\sigma$ always just steps to $c$ ). It does, however, indicate a program point where type checking should occur. Hence, we can independently typecheck $c$ at type $\sigma$ and then use $\sigma$ when checking the rest of the program.

Intuition for П4's type system. Next, we give an intuition for П4's types. A command $c$ is always assigned a dependent function type $\left(x: \tau_{1}\right) \rightarrow \tau_{2}$, where $x$ may occur in $\tau_{2}$. This design allows us to relate the input and output values of commands expressed in the heap types $\tau_{1}$ and $\tau_{2}$. For example, we may want to ensure that the Ethernet header has the same value after being deparsed, reset, and then reparsed. To express equations like this, we use refinement types $\{y: \tau \mid \varphi\}$, where $\varphi$ is a formula in the logic of variable-width bit vectors with concatenation and length operators. In this example, we could say that the Ethernet header is unchanged by using the output type $\left\{y: \tau_{2} \mid x\right.$. eth $=y$.eth $\}$.

We also often need to reference intermediate formulae, so we introduce substitution types, written $\tau_{2}\left[x \mapsto \tau_{1}\right]$, where $x$ may occur in $\tau_{2}$ but not in $\tau_{1}$. In such a type, $\tau_{1}$ may represent the type at any earlier point in the program. $\Pi 4$ also supports fine-grained path-dependent reasoning via union types $\left(\tau_{1}+\tau_{2}\right)$. It is also convenient to have trivial $(T)$ and absurd $(\varnothing)$ types.

\footnotetext{
${ }^{2} \mathrm{P} 4$ allows loops within parsers, but because programs are restricted to finite state, the language specification allows implementations to unroll loops.

${ }^{3}$ The difference here is similar to the difference between C's malloc (analogous to P4's semantics) and calloc (analogous to П4's semantics).
} 


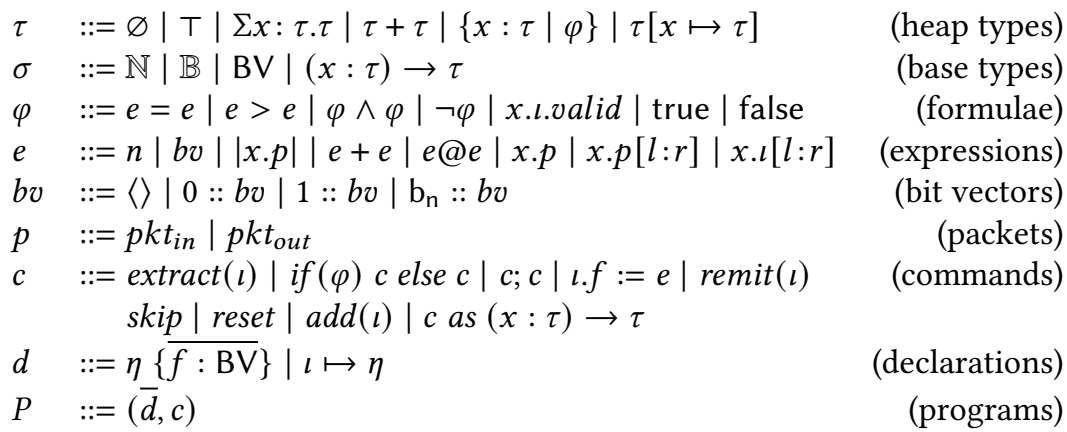

Fig. 3. Syntax of $\Pi 4$

Finally, the design of our type system is also informed by the need to model parsing operations. Specifically, we must ensure that the refinements on the input type and on the output type remain consistent after bits have been shuffled around by a command. For example, given an input type $\left\{x: \top\left|x \cdot p k t_{i n}[0: 8]==4 \wedge\right| x \cdot p k t_{i n}|>| i p v 4 \mid\right\}$, where $x . p k t_{\text {in }}$ represents the incoming packet, and the command extract(ipv4), the output type should reflect that the ipv4 header instance is now valid, that ipv $4[0: 8]$ is 4 , and that $x$.pkt $t_{\text {in }}$ may have no more bits remaining. $\Pi 4$ accomplishes this using two key mechanisms: (1) a dependent sum type $\Sigma x: \tau_{1} \cdot \tau_{2}$ that computes the disjoint union of the valid instances in $\tau_{1}$ and $\tau_{2}$ and concatenates the incoming and outgoing packets (Section 3.3), and (2) a refinement transformer, chomp, that manipulates input refinements to be consistent with the extraction operation (see Section 3.5).

\subsection{Syntax}

Figure 3 shows the syntax of $\Pi 4$. Boolean formulae $\varphi$ include literals, equality $(=)$ and validity of instances (x.l.valid), conjunction $(\wedge)$, and negation $(\neg)$. Expressions $e$ include naturals, bit vectors, packet length $(|x . p|)$, addition $(+)$, concatenation (@), packet access (x.p) and slices of packets $(x . p[l: r])$ and instances $(x . \iota[l: r])$.

To ease the notation, we write $x . l[l]$ instead of $x . l[l: l+1]$ for bit-wise access, $x . l . f$ instead of $x . l[l: r]$ for ranges matching header instance fields, $x . l$ instead of $x . l[0: \operatorname{sizeof}(\iota)], x . l[n:]$ for the remaining bits of $x . \iota$ starting from bit $n+1$, and similarly for the corresponding formulae involving packet variables $x$.p. We use a list-like encoding of bit vectors. A bit vector is either the empty bit vector $(\langle\rangle)$ or a concatenation of bits. We assume that bit variables $b_{n}$ are not part of the surface syntax and are only used internally. For singleton bit vectors, we write $\langle b\rangle$ instead of $b::\langle\rangle$.

We write $\epsilon \triangleq\left\{x: \top \mid \bigwedge_{\iota \in \operatorname{dom}(\mathcal{H} \mathcal{T})} \neg x . \iota . v a l i d\right\}$ for the type denoting the empty heap on which no header instances are valid, $\iota \triangleq\left\{x: \top \mid x . \iota . v a l i d \wedge \bigwedge_{\iota^{\prime} \in \operatorname{dom}(\mathcal{H} \mathcal{T}), \iota^{\prime} \neq l} \neg x . \iota^{\prime}\right.$. valid $\}$ for the type denoting the heap exclusively containing instance $\iota$, and $\iota \sim \triangleq x: \top \mid x . l . v a l i d\}$ for the type denoting the heap on which at least instance $\iota$ is guaranteed to be valid.

For formulae, we write $x \equiv y$ (respectively $x \equiv, y$ ) as syntactic sugar for the boolean predicates capturing strict equality (respectively instance equality) between the heaps bound to $x$ and $y$. Strict equality requires that both the input and output packets are equivalent as well as all instances contained in the heap-i.e., $x . p k t_{\text {in }}=y \cdot p k t_{\text {in }} \wedge x \cdot p k t_{\text {out }}=y \cdot p k t_{\text {out }} \wedge \wedge_{\iota \in \operatorname{dom}(\mathcal{H} \mathcal{T})} x . \iota=y \cdot l$, while instance equality only requires that the instances are equivalent in both heaps. We write $x$.l.valid $=$ y.ı.valid as syntactic sugar for (x.l.valid $\wedge$ y.ı.valid $) \vee(\neg$ x.ı.valid $\wedge \neg$ y.ı.valid $)$. We use standard encodings using negation and conjunction for logical connectives like $\vee$ or $\Rightarrow$. 


$$
\begin{aligned}
\llbracket \tau \rrbracket_{\mathcal{E}} & \subseteq \mathcal{H} \\
\llbracket \varnothing \rrbracket_{\mathcal{E}} & =\{\} \\
\llbracket \top \rrbracket_{\mathcal{E}} & =\mathcal{H} \\
\llbracket \tau_{1}+\tau_{2} \rrbracket_{\mathcal{E}} & =\llbracket \tau_{1} \rrbracket_{\mathcal{E}} \cup \llbracket \tau_{2} \rrbracket_{\mathcal{E}} \\
\llbracket \Sigma x: \tau_{1} \cdot \tau_{2} \rrbracket_{\mathcal{E}} & =\left\{h_{1}+h_{2} \mid h_{1} \in \llbracket \tau_{1} \rrbracket_{\mathcal{E}} \wedge h_{2} \in \llbracket \tau_{2} \rrbracket_{\mathcal{E}\left[x \mapsto h_{1}\right]}\right\} \\
\llbracket \tau_{1}\left[x \mapsto \tau_{2}\right] \rrbracket_{\mathcal{E}} & =\left\{h \mid h_{2} \in \llbracket \tau_{2} \rrbracket_{\mathcal{E}} \wedge h \in \llbracket \tau_{1} \rrbracket_{\mathcal{E}\left[x \mapsto h_{2}\right]}\right\} \\
\llbracket\{x: \tau \mid \varphi\} \rrbracket_{\mathcal{E}} & =\left\{h \mid h \in \llbracket \tau \rrbracket_{\mathcal{E}} \wedge \llbracket \varphi \rrbracket \mathcal{E}[x \mapsto h]=\text { true }\right\}
\end{aligned}
$$

Fig. 4. Semantics of heap types

We write $\bar{x}$ as a shorthand for a possibly empty sequence $x_{1}, \ldots, x_{n}$. A program consists of a sequence of declarations $\bar{d}$ and a command $c$. Declarations $d$ include header type declarations $\eta\{\overline{f: \mathrm{BV}}\}$ and header instance declarations $\iota \mapsto \eta$. Header type declarations specify the format of network packet headers. They are defined in terms of a name and a sequence of field declarations, where each field is itself defined in terms of a field name and a type. We write $f$ : BV to denote that field $f$ has a bit vector type BV. With $\eta$ ranging over header types, the instance declaration $\iota \mapsto \eta$ assigns the name $\iota$ to header type $\eta$. The global mapping between header instances and header types is stored in the so-called header table $\mathcal{H} \mathcal{T}$. We assume that names of header instances and header types are drawn from disjoint sets of names and that each name is declared only once.

$\Pi 4$ provides commands for parsing (extract), creating ( $a d d)$ and modifying $(\iota . f:=t)$ header instances. The remit command serializes a header instance into a bit sequence. The reset command resets the program state-in particular, the packet buffers. Commands can be sequentially composed $\left(c_{1} ; c_{2}\right)$, skip is a no-op, and the if-command conditionally executes one out of two commands based on the value of the boolean formula $\varphi$. We assume that formulae and expressions used in commands are implicitly prefixed with a variable named heap, but we often omit it in the surface syntax. For example, we write if (ether.valid) then extract(ipv4) else skip instead of if (heap.ether.valid) then extract (heap.ipv4) else skip. $\Pi 4$ provides modular reasoning via type ascription $\left(c\right.$ as $\left.\left(x: \tau_{1}\right) \rightarrow \tau_{2}\right)$. Finally, we assume that every header referenced in a program has a corresponding instance declaration-this could be enforced statically using a simple analysis.

\subsection{Type System}

As shown in Figure 3, there are two categories of types, base types $\sigma$ and heap types $\tau$. Base types include natural numbers, bit vectors, booleans, and dependent function types. Heap types $\tau$ represent sets of heaps, where each element in the set describes a different program path. The goal is to capture bit-level dependencies between header instances and the incoming and outgoing packet in the type system. A heap $h$ in the set of heaps $\mathcal{H}$ describes a possible system state, consisting of the incoming and outgoing packet and the set of valid header instances. We model heaps as maps from names to bit vectors. A heap contains two special entries $p k t_{\text {in }}$ and $p k t_{\text {out }}$ representing the incoming and outgoing packet buffers, as well as mappings from instance names to bit vectors for each valid header instance. The semantics of types is shown in Figure 4. Heap types are evaluated in an environment $\mathcal{E}$, which is a mapping from variable names to heaps. The environment models other heaps available in the current scope upon which the current header type may depend.

The type $\varnothing$ denotes the empty set. It is used in situations where there are unsatisfiable assumptions involving the header instances or the incoming and outgoing packet buffers. 


$$
\begin{aligned}
& \llbracket e \rrbracket_{\mathcal{E}} \in \mathrm{B} \vee \cup \mathbb{N} \\
& \llbracket|x . p| \rrbracket_{\mathcal{E}}= \begin{cases}0 & \text { if } \mathcal{E}(x)(p)=\langle\rangle \\
n & \text { if } \mathcal{E}(x)(p)=\left\langle b_{1}, \ldots, b_{n}\right\rangle \\
\perp & \text { otherwise }\end{cases} \\
& \llbracket \llbracket x . p \rrbracket_{\mathcal{E}}= \begin{cases}\left\langle b_{1}, \ldots, b_{n}\right\rangle & \text { if } \mathcal{E}(x)(p)=\left\langle b_{1}, \ldots, b_{n}\right\rangle \\
\perp & \text { otherwise }\end{cases} \\
& \llbracket x . p[n: m] \rrbracket_{\mathcal{E}}= \begin{cases}\left\langle b_{n}, \ldots, b_{m-1}\right\rangle & \text { if } \llbracket x . p \rrbracket_{\mathcal{E}}=\left\langle b_{0}, \ldots, b_{k}\right\rangle \wedge \\
\perp & 0 \leq n<m \leq k+1\end{cases} \\
& \llbracket x . l[n: m] \rrbracket_{\mathcal{E}}= \begin{cases}\left\langle b_{n}, \ldots, b_{m-1}\right\rangle & \text { if } \llbracket x . l \rrbracket_{\mathcal{E}}=\left\langle b_{0}, \ldots, b_{k}\right\rangle \wedge \\
\perp & 0 \leq n<m \leq k+1\end{cases}
\end{aligned}
$$

Fig. 5. Selected cases of the semantics of expressions

The top type $T$ denotes the set of all possible heaps. The choice type $\tau_{1}+\tau_{2}$ denotes the union of the sets of heaps represented by $\tau_{1}$ and $\tau_{2}$. The dependent pair $\Sigma x: \tau_{1} \cdot \tau_{2}$ denotes the concatenation of heaps from $\tau_{1}$ and $\tau_{2}$, where heaps described by $\tau_{2}$ may depend on heaps from $\tau_{1}$. The concatenation $h=h_{1}+h_{2}$ of two heaps $h_{1}$ and $h_{2}$ requires that header instances contained in $h_{1}$ and $h_{2}$ are disjoint. The resulting heap contains all instances from $h_{1}$ and from $h_{2}$, with $p k t_{\text {in }}$ and $p k t_{\text {out }}$ the concatenation of respective bit vectors in $h_{1}$ and $h_{2}$. The explicit substitution $\tau_{1}\left[x \mapsto \tau_{2}\right]$ denotes the set of heaps obtained by evaluating $\tau_{1}$ for every heap described by $\tau_{2}$. Finally, the refinement type $\{x: \tau \mid e\}$ denotes the set of heaps described by $\tau$ for which the predicate $e$ holds.

The refinement predicate $\varphi$ is evaluated in the same type of environment as heap types. Formulae evaluate to a boolean value, i.e., $\llbracket \varphi \rrbracket_{\mathcal{E}} \in \mathbb{B}$. The semantics of expression equality $\left(e_{1}=e_{2}\right)$ is defined as the semantic equality between expressions $e_{1}$ and $e_{2}$. If the semantics of one of the expressions is undefined, expression equality always evaluates to false. The semantics of expression comparison $\left(e_{1}>e_{2}\right)$ is defined analogously. Instance validity $x$.ı. valid evaluates to true if header instance $\iota$ is contained in the heap bound to $x$ in $\mathcal{E}$, otherwise it evaluates to false. The remaining operations have standard semantics.

The semantics of expressions is defined in Figure 5. For brevity, we omit standard cases. Expressions evaluate to either a bit vector or a natural number. For the semantic addition (+) and bit vector concatenation operator (@), we assume that as soon as evaluating one operand results in an error $(\perp)$ the whole expression also evaluates to $\perp$. To evaluate the length of a packet $|x . p|$, we compute the length of the bit vector of $p k t_{\text {in }}$ or $p k t_{\text {out }}$ respectively in the heap bound to $x$ in the environment. If no heap is bound to variable name $x$, the expression evaluates to $\perp$. The semantics of bit vectors is as expected, except for variables, which we look up from a designated location in the environment. If no binding for the bit variable exists, it evaluates to $\perp$. The semantics of bit vector concatenation is standard. A packet access $x$.p looks up the respective entry from the heap bound to variable $x$ in $\mathcal{E}$. A packet slice $x . p[l: r]$ is evaluated in the same way, but additionally the designated slice is obtained from the bit vector. Again, if the variable is not bound or an index is greater than the length of the bit vector, the expression evaluates to $\perp$. The semantics of instance slices $x . l[l: r]$ is defined similarly but the lookup occurs on header instance $\iota$. We interpret slices as 


$$
\begin{aligned}
& \text { E-ExTract } \\
& \qquad \mathcal{H} \mathcal{T}(\iota)=\eta \quad \text { deserialize }_{\eta}(I)=\left(v, I^{\prime}\right) \\
& \langle I, O, H, \text { extract }(\iota)\rangle \rightarrow\left\langle I^{\prime}, O, H[\iota \mapsto v], \text { skip }\right\rangle
\end{aligned}
$$$$
\text { E-MoD }
$$

$$
\frac{H(\iota)=r \quad r^{\prime} \triangleq\{r \text { with } f=v\}}{\langle I, O, H, \iota . f:=v\rangle \rightarrow\left\langle I, O, H\left[\iota \mapsto r^{\prime}\right], \text { skip }\right\rangle}
$$

E-ADD

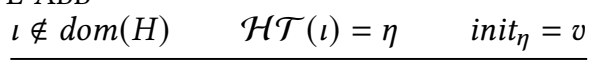

\section{E-REMIT}

$$
\begin{gathered}
\iota \in \operatorname{dom}(H) \quad \mathcal{H} \mathcal{T}(\iota)=\eta \\
\operatorname{serialize}_{\eta}(H(\iota))=b v \\
\langle I, O, H, \text { remit }(\iota)\rangle \rightarrow\langle I, O:: b v, H, \text { skip }\rangle
\end{gathered}
$$

E-RESET

$$
\frac{I^{\prime}=O @ I}{\langle I, O, H, \text { reset }\rangle \rightarrow\left\langle I^{\prime},\langle\rangle,[], \text { skip }\right\rangle}
$$

\section{E-AsCRIBE}

$$
\overline{\left\langle I, O, H, c \text { as }\left(x: \tau_{1}\right) \rightarrow \tau_{2}\right\rangle \rightarrow\langle I, O, H, c\rangle}
$$

Fig. 6. Small-step operational semantics of $\Pi 4$

half-open intervals, where the left bound is included and the right bound is excluded. For example, given a bit vector $b v=1010$ we have $b v[1: 4]=010$.

We define two semantic operations on heap types: inclusion and exclusion of instances. The first, Includes $\Gamma \tau \iota$, traverses $\tau$ and checks that instance $\iota$ is valid in every path. Semantically this says that $\iota$ is a member of every element of $\llbracket \tau \rrbracket_{\mathcal{E}}-$ i.e., if $\mathcal{E} \vDash \Gamma$, then $\forall h \in \llbracket \tau \rrbracket_{\mathcal{E} . l} \in \operatorname{dom}(h)$. The second, Excludes $\Gamma \tau \iota$, traverses $\tau$ and checks that instance $\iota$ is invalid in every path. Semantically this says that $\iota$ is no member of every element of $\llbracket \tau \rrbracket_{\mathcal{E}}$-i.e., if $\mathcal{E} \vDash \Gamma$, then $\forall h \in \llbracket \tau \rrbracket_{\mathcal{E} . \iota} \notin \operatorname{dom}(h)$.

\subsection{Operational Semantics}

The small-step operational semantics of $\Pi 4$, is shown in Figure 6. It is defined in terms of a four-tuple $\langle I, O, H, c\rangle$, where $I$ and $O$ are the bitstrings for the incoming and outgoing packets respectively, $H$ is a map that relates instance names to records containing the field values, and $c$ is a command.

The extract $(\iota)$ command (E-ExтRACT) first looks up the header type from the header table $(\mathcal{H} \mathcal{T})$, and uses a deserialization function to copy the appropriate number of bits from the input packet into the deserialized representation of the instance $v$. This value is added to the map of valid header instances $H$. We assume there exists a deserialization function for every header instance. For example, assuming $I=110011 B$, where $B$ is the rest of the bitstring, and $\eta=\{f: 4 ; g: 2\}$, then deserialize $_{\eta}(I)=(\{f=1100 ; g=11\}, B)$.

The remit $(\iota)$ command (rule E-REMIT) requires that the header instance is valid-i.e., it is contained in $H$. Similar to E-ExтRACT, we assume there is a serialization function for every heap type, which turns a record representing the instance back into a bit sequence. For example, serialize $_{\eta}(\{f=1100 ; g=11\})=11011$. The serialized bit sequence is appended to the end of the outgoing packet. Both the input packet and the set of valid headers remain unchanged.

The rule E-MoD defines the semantics of assigning a value to a header field. Assuming $r$ is the record storing the values of the fields, an updated record $r^{\prime}$ with the modified field value is stored in $H$. The input and output packets remain unchanged. If the assigned expression is not a value, it is reduced first.

The rules for sequencing (E-SEQ, E-SEQ1) are standard. Sequences of commands evaluate from left to right-i.e., the left-hand command is reduced to skip before the right-hand command is evaluated. The evaluation rules for conditionals (E-IF, E-IFTrue, E-IFFALSE) are also standard. All standard rules are omitted. 
The rule E-RESET defines the semantics of the command reset. It would be invoked between the ingress and egress pipelines, when the packet emitted by the ingress becomes the input packet for the egress. Operationally, the bits contained in the output packet are prepended to the bits of the input packet. This concatenated bit sequence serves as the new input packet. The output packet is emptied and all valid header instances are discarded.

The rule E-ADD initializes a header instance if it is not already valid. The evaluation is similar to rule E-ExтRAст, except that no bits are taken from the input bitstring. Instead we assume that there exists an initialization function init $_{\eta}$ for every heap type $\eta$-similar to deserialize-that initializes all fields of an instance to a fixed value. If an instance is already valid, this operation is a no-op. An ascribed command $c$ as $\sigma$ (rule E-AscriBE) evaluates to $c$ trivially, without modifying the heap.

\subsection{Typing Judgement}

The typing judgement has the form $\Gamma \vdash c:\left(x: \tau_{1}\right) \rightarrow \tau_{2}$. Intuitively, type $\tau_{1}$ describes the input heap and $\tau_{2}$ describes the output heap obtained after the execution of $c . \Gamma$ is a variable context that maps variable names to heap types and is used to capture additional dependencies of the input type. If a command typechecks in a context where $x$ maps to $\tau$ (i.e., $\left.\Gamma, x: \tau \vdash c:\left(x: \tau_{1}\right) \rightarrow \tau_{2}\right)$ it means that given some heap described by type $\tau$ on which the input heap might depend, executing $c$ on the input heap described by $\tau_{1}$ will result in a heap described by $\tau_{2}$.

The typing rules are presented in Figure 7. The typing rule T-ExTRACT captures that $\iota$ must be valid after an extract command is executed and the input packet of type $\tau_{1}$ provides enough bits for the instance $\left(\operatorname{sizeof}_{p k t_{i n}}(\tau) \geq n\right.$ iff $\forall \mathcal{E}, h \in \llbracket \tau \rrbracket_{\mathcal{E}},\left|h\left(p k t_{i n}\right)\right| \geq n$ ). Intuitively, the chomp operator ensures that the output type reflects that the first $n$ bits, where $n$ is the number of bits contained in header instance $l$, are removed from the input packet and copied into instance $l$.

The typing rule for sequencing T-SEQ is mostly standard, with one peculiarity: because our typing judgement assigns dependent function types to commands, the result type $\tau_{22}$ of command $c_{2}$ might depend on its input type $\tau_{12}-$ i.e., variable $y$ might appear free in $\tau_{22}$. Hence, we must also capture the type $\tau_{12}$ in the result type. The typing rule T-SKIP is standard, except that it strictly enforces that the heaps described by the output type are equivalent to the heaps described by the input type. To typecheck the command remit, we check whether the instance to be emitted is guaranteed to be valid in the input type. The assigned output type ensures that emitting a header instance appends the value of the instance to the end of the outgoing packet (second projection of the assigned $\Sigma$-type) but leaves the input packet and all other validity information unchanged (first projection of the assigned $\Sigma$-type). The rule T-RESET resets all assumptions about header validity, empties $p k t_{\text {out }}$ and refines $p k t_{\text {in }}$ to be the concatenation of $p k t_{\text {out }}$ and $p k t_{\text {in }}$ of the input type. In the output type, we use a $\Sigma$-type to model the concatenation.

The rule T-IF typechecks each branch of the conditional with the additional assumption that the condition $\varphi$ holds respectively does not hold. The resulting type is a path-sensitive union type, which includes the types of both paths. By default, all variables in formula $\varphi$ in the command are bound to heap. To turn $\varphi$ into a refinement on a type, we substitute every occurrence of heap with the respective binder of the type we want to refine. We write $\varphi[x /$ heap $]$ to denote the formula obtained from $\varphi$ in which heap is replaced with $x$. For example, if the command is if (ether.etherType $=0 \times 0800$ ) then extract (ipv4) else skip, we typecheck the then-branch with type $\left(x:\left\{y: \tau_{1} \mid y\right.\right.$.ether.etherType $\left.\left.=0 \times 0800\right\}\right) \rightarrow \tau_{12}$. The full command is checked with type $\left(x: \tau_{1}\right) \rightarrow\left\{y: \tau_{12} \mid x\right.$.ether.etherType $\left.=0 \times 0800\right\}+\left\{y: \tau_{22} \mid \neg x\right.$.ether.etherType $\left.=0 \times 0800\right\}$.

To typecheck a modification of an instance field, the typing rule T-MoD first checks if the instance to be modified is guaranteed to be valid in the input type. The output type is similar to the strongestpostcondition of the input type: everything in the output type is the same as in $x$, except for the modified instance field $y . l . f$, which must be equal to $e[x /$ heap $]$. 


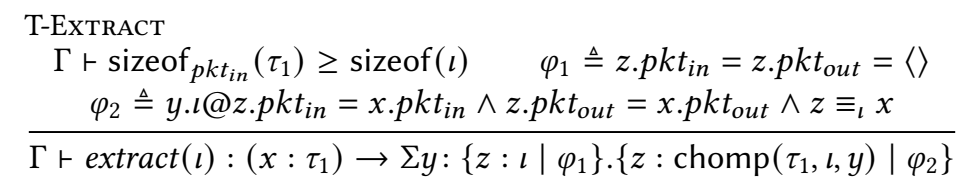

T-SEQ

$$
\begin{aligned}
& \Gamma \vdash c_{1}:\left(x: \tau_{1}\right) \rightarrow \tau_{12} \\
& \frac{\Gamma, x: \tau_{1} \vdash c_{2}:\left(y: \tau_{12}\right) \rightarrow \tau_{22}}{\Gamma \vdash c_{1} ; c_{2}:\left(x: \tau_{1}\right) \rightarrow \tau_{22}\left[y \mapsto \tau_{12}\right]} \quad \frac{\tau_{2} \triangleq\left\{y: \tau_{1} \mid y \equiv x\right\}}{\Gamma \vdash \text { skip }:\left(x: \tau_{1}\right) \rightarrow \tau_{2}} \\
& \text { T-Remit } \\
& \frac{\text { Includes } \Gamma \tau_{1} \iota \quad \varphi \triangleq z \cdot p k t_{\text {in }}=\langle\rangle \wedge z \cdot p k t_{\text {out }}=x . \iota}{\Gamma \vdash \operatorname{remit}(\iota):\left(x: \tau_{1}\right) \rightarrow \Sigma y:\left\{z: \tau_{1} \mid z \equiv x\right\} .\{z: \epsilon \mid \varphi\}}
\end{aligned}
$$

T-RESET

$$
\begin{array}{cc}
\varphi_{1} \triangleq z \cdot p k t_{\text {out }}=\langle\rangle \wedge z \cdot p k t_{\text {in }}=x \cdot p k t_{\text {out }} & \text { T-AsCRIBE } \\
\varphi_{2} \triangleq z \cdot p k t_{\text {out }}=\langle\rangle \wedge z \cdot p k t_{\text {in }}=x \cdot p k t_{\text {in }} & \Gamma \vdash c: \sigma \\
\hline \Gamma \vdash \text { reset }:\left(x: \tau_{1}\right) \rightarrow \Sigma y:\left\{z: \epsilon \mid \varphi_{1}\right\} \cdot\left\{z: \epsilon \mid \varphi_{2}\right\} & \frac{\Gamma \vdash c \text { as } \sigma: \sigma}{\Gamma \vdash \delta}
\end{array}
$$

T-IF

$$
\begin{gathered}
\Gamma \vdash \tau \vdash \varphi: \mathbb{B} \\
\Gamma \vdash c_{1}:\left(x:\left\{y: \tau_{1} \mid \varphi[y / \text { heap }]\right\}\right) \rightarrow \tau_{12} \quad \Gamma \vdash c_{2}:\left(x:\left\{y: \tau_{1} \mid \neg \varphi[y / \text { heap }]\right\}\right) \rightarrow \tau_{22} \\
\Gamma \vdash \operatorname{if}(\varphi) c_{1} \text { else } c_{2}:\left(x: \tau_{1}\right) \rightarrow\left\{y: \tau_{12} \mid \varphi[x / \text { heap }]\right\}+\left\{y: \tau_{22} \mid \neg \varphi[x / \text { heap }]\right\}
\end{gathered}
$$

T-MOD

$$
\begin{aligned}
& \text { Includes } \Gamma \tau_{1} \iota \\
& \mathcal{F}(\iota, f)=\mathrm{BV} \quad ; \tau_{1} \vdash e: \mathrm{BV} \quad \varphi_{p k t} \triangleq y \cdot p k t_{\text {in }}=x \cdot p k t_{\text {in }} \wedge y \cdot p k t_{\text {out }}=x \cdot p k t_{\text {out }}
\end{aligned}
$$

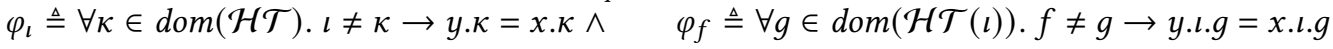

$$
\begin{aligned}
& \Gamma \vdash \iota . f:=e:\left(x: \tau_{1}\right) \rightarrow\left\{y: \top \mid \varphi_{p k t} \wedge \varphi_{\iota} \wedge \varphi_{f} \wedge y . l . f=e[x / \text { heap }]\right\} \\
& \text { Excludes } \Gamma \tau_{1} \iota \quad \text { init }_{\mathcal{H} \mathcal{T}(l)}=v \\
& \frac{\varphi \triangleq z \cdot p k t_{\text {in }}=z \cdot p k t_{\text {out }}=\langle\rangle \wedge z \cdot \iota=v}{\Gamma \vdash \operatorname{add}(\iota):\left(x: \tau_{1}\right) \rightarrow \Sigma y:\left\{z: \tau_{1} \mid z \equiv x\right\} \cdot\{z: \iota \mid \varphi\}} \\
& \Gamma \vdash \tau_{1}<: \tau_{3} \\
& \Gamma, x: \tau_{1} \vdash \tau_{4}<: \tau_{2} \\
& \frac{\Gamma \vdash c:\left(x: \tau_{3}\right) \rightarrow \tau_{4}}{\Gamma \vdash c:\left(x: \tau_{1}\right) \rightarrow \tau_{2}}
\end{aligned}
$$

Fig. 7. Command typing rules for $\Pi 4$.

Rule T-ADD first checks that the instance is not yet included in the type and assigns an output type that reflects that all information from the input type $\tau_{1}$ are retained and just instance $\iota$ is added. The typing rule for ascription T-AsCRIBE is standard. The typing rule for subsumption T-SuB is also standard. We write $\Gamma \vdash \tau_{1}<: \tau_{2}$ to denote the subtyping check between $\tau_{1}$ and $\tau_{2}$. The contexts $\Gamma_{1}$ and $\Gamma_{2}$ capture external dependencies of $\tau_{1}$ and $\tau_{2}$ respectively.

We take a semantic approach for defining subtyping as shown in the left of Figure 8. Type $\tau_{1}$ with context $\Gamma_{1}$ is a subtype of type $\tau_{2}$ with context $\Gamma_{2}$, if and only if for all environments $\mathcal{E}_{1}$ and $\mathcal{E}_{2}$ such that $\mathcal{E}_{1}$ entails the context $\Gamma_{1}$ for subtype $\tau_{1}$ and $\mathcal{E}_{2}$ entails the context $\Gamma_{2}$ for supertype $\tau_{2}$, the 


$$
\Gamma \vdash \tau_{1}<: \tau_{2} \stackrel{\Delta}{\Leftrightarrow} \forall \mathcal{E} \vDash \Gamma . \llbracket \tau_{1} \rrbracket_{\mathcal{E}} \subseteq \llbracket \tau_{2} \rrbracket_{\mathcal{E}} \quad \mathcal{E} \vDash \Gamma \stackrel{\Delta}{\Leftrightarrow} \forall x_{i} \in \operatorname{dom}(\Gamma) . \mathcal{E}\left(x_{i}\right)=h_{i} \wedge h_{i} \models_{\mathcal{E}} \Gamma\left(x_{i}\right)
$$

Fig. 8. Left: Subtyping. Right: Entailment between environments and subtyping contexts.

\begin{tabular}{|c|c|c|c|}
\hline ENT-ToP & $\begin{array}{l}\text { ENT-ChOICEL } \\
\qquad(I, O, H) \models_{\mathcal{E}} \tau_{1}\end{array}$ & $\begin{array}{l}\text { Ent-ChOICER } \\
\quad(I, O, H) \models_{\mathcal{E}} \tau_{2}\end{array}$ & $\begin{array}{l}\text { ENT-REFINE } \\
\qquad(I, O, H) \models_{\mathcal{E}} \tau \\
\llbracket \varphi \rrbracket_{\mathcal{E}[x \mapsto(I, O, H)]=\text { true }}\end{array}$ \\
\hline$(I, O, H) \models_{\mathcal{E}} \top$ & $\overline{(I, O, H) \vDash \mathcal{E} \tau_{1}+\tau_{2}}$ & $\overline{(I, O, H) \models_{\mathcal{E}} \tau_{1}+\tau_{2}}$ & $(I, O, H) \vDash_{\mathcal{E}}\{x: \tau \mid \varphi\}$ \\
\hline \multicolumn{2}{|c|}{$\begin{array}{l}\text { ENT-Sigma } \\
\qquad \begin{array}{c}\left(I_{1}, O_{1}, H_{1}\right) \models_{\mathcal{E}} \tau_{1} \\
\left(I_{2}, O_{2}, H_{2}\right) \models_{\mathcal{E}\left[x \mapsto\left(I_{1}, O_{1}, H_{1}\right)\right]} \tau_{2} \\
\end{array}\end{array}$} & \multicolumn{2}{|c|}{$\begin{array}{l}\text { EnT-SuBST } \\
\qquad \begin{array}{c}\left(I_{2}, O_{2}, H_{2}\right) \models_{\mathcal{E}} \tau_{2} \\
(I, O, H) \models \mathcal{E}\left[x \mapsto\left(I_{2}, O_{2}, H_{2}\right)\right] \tau_{1} \\
\end{array}\end{array}$} \\
\hline \multicolumn{4}{|c|}{$\left(I_{1} @ I_{2}, O_{1} @ O_{2}, H_{1} \cup H_{2}\right) \models_{\mathcal{E}} \Sigma x: \tau_{1} \cdot \tau_{2}$} \\
\hline
\end{tabular}

Fig. 9. Entailment between heaps and heap types.

set of heaps described by $\tau_{1}$ evaluated in environment $\mathcal{E}_{1}$ is a subset of the set of heaps described by $\tau_{2}$ evaluated in environment $\mathcal{E}_{2}$.

The entailment between environments and typing contexts is defined in the right of Figure 8 . An environment $\mathcal{E}$ entails a context $\Gamma$, iff for every mapping from a variable name $x_{i}$ to some heap type $\tau_{i}$ in $\Gamma$ there exists a mapping from variable $x_{i}$ to some heap $h_{i}$ in environment $\mathcal{E}$ and that heap $h_{i}$ entails type $\tau_{i}$. The entailment between a heap and a type is defined in Figure 9. A heap $H\left[p k t_{\text {in }} \mapsto I, p k t_{\text {out }} \mapsto O\right]$, in short $(I, O, H)$ entails a type $\tau$, if it is contained in the type.

\subsection{Chomp}

When an instance $\iota$ is extracted, sizeof $(\iota)$ bits are moved from the input bitstring to the instance-we call this chomping. To reflect it in the type that we assign to an extract command, we define a syntactic operation chomp that transforms a heap type into the heap type that would result from extracting an instance.

We first specify a semantic chomp operation on a single heap $\left(\operatorname{chomp}^{\Downarrow}(h, n)\right)$ in Definition 3.1-it removes the first $n$ bits from the input packet in heap $h$.

Definition 3.1 (Semantic Chomp). chomp ${ }^{\Downarrow}(h, n)=h\left[p k t_{\text {in }} \mapsto h\left(p k t_{\text {in }}\right)[n:]\right]$

Intuitively, syntactic chomp lifts chomp ${ }^{\Downarrow}$ to heap types (written formally in Lemma 3.2). For example, given a header instance $\mathrm{A}$ of type $\mathrm{A}_{-} \mathrm{t}\{\mathrm{f}: 2\}$, $\operatorname{chomp}\left(\left\{x: \epsilon \mid x \cdot p k t_{\text {in }}[0: 2]=\right.\right.$ $11\}, A, y)=\{x: \epsilon \mid y \cdot A[0: 2]=11\}$, i.e., because header instance $\mathrm{A}$ contains two bits, the first two bits are moved from $p k t_{\text {in }}$ to instance A, bound by $y$. It turns out that we can define chomp via a simple syntactic transformation. To do this, we first define a single-bit operation, chomp $_{1}$, that processes only a single bit. Then chomp recursively lifts chomp $p_{1}$ to the appropriate length.

3.6.1 Chomp ${ }_{1}$. To chomp a single bit from a heap type, we will need to update all references to the length, as well as to the first bit of $p k t_{i n}$. This computation resembles Brzozowski derivatives [Brzozowski 1964]. For the complete definition of chomp ${ }_{1}$, we refer the reader to the companion technical report. Here we provide some intuition for how it works. Semantically, chomp ${ }_{1}\left(\tau, \mathrm{b}_{0}\right)$ transforms for each heap $h$ denoted by a heap type $\tau$, into the heap $h\left[p k t_{\text {in }} \mapsto h\left(p k t_{\text {in }}\right)[1:]\right]$. The variable $\mathrm{b}_{0}$ is a placeholder corresponding to the missing bit. Then, a helper function heapRef ${ }_{1}$ replaces the 
placeholder bits introduced by chomp ${ }_{1}$ with references to the extracted bit. In particular, the $i$-th call to heapRef ${ }_{1}$, replaces variable $b_{i}$ with $x . l[i-1: i]$.

Syntactically, when chomping a heap type $\tau$ we update each occurrence of $p k t_{i n}$ in a refinement, if that occurrence describes the first bit of $p k t_{i n}$ of a heap in the semantics of $\tau$. Types $\varnothing$ and T are not affected by chomping. For a choice type $\tau_{1}+\tau_{2}$, chomp $p_{1}$ is applied to both $\tau_{1}$ and $\tau_{2}$ individually, as each branch of the choice type describes isolated heaps of $\tau$. In the substitution type, $\tau_{1}\left[x \mapsto \tau_{2}\right]$, only $\tau_{1}$ is chomped, as $\tau_{2}$ only captures information relevant for evaluating refinements.

For the refinement type $\left\{x: \tau_{1} \mid \varphi\right\}$, chomp is applied recursively to $\tau_{1}$ and all references to the first bit of $p k t_{i n}$ as well as the length of $p k t_{i n}$ are updated accordingly. We increment numeric expressions referencing $x$.pkt $t_{i n}$ (e.g. the refinement $\left|x . p k t_{i n}\right|$ becomes $\left|x . p k t_{i n}\right|+1$ and we prepend the placeholder bit $b_{\mathrm{n}}$ for bit-vector expressions referencing $x . p k t_{i n}$ ).

When we apply chomp $p_{1}$ to type $\Sigma x: \tau_{1} \cdot \tau_{2}$, we have to distinguish two cases, either the input packet described by $\tau_{1}$ contains at least one bit or the input packet described by $\tau_{1}$ is empty. In the first case, chomp $p_{1}$ removes the first bit of $p k t_{i n}$ in $\tau_{1}$ and in the second case it removes the first bit of $p k t_{i n}$ in $\tau_{2}$. If we chomp in $\tau_{1}$ we need to update all refinements to $x$.pkt $t_{i n}$ in $\tau_{2}$, as $\tau_{1}$ is bound to $x$ in $\tau_{2}$; otherwise chomping could cause contradictions between refinements referencing the same component. Similar to the computation of a Brzozowski derivative of a product, the result is the union of the type obtained by chomping $\tau_{1}$ and $\tau_{2}$ respectively, where we additionally assert in the second case that $p k t_{\text {in }}$ of $\tau_{1}$ must be empty.

Example. Given type $\tau=\Sigma x:\left\{y: \epsilon|| y \cdot p k t_{\text {in }} \mid=1\right\} .\left\{z: \epsilon|| x \cdot p k t_{\text {in }} \mid=1\right\}$

$$
\begin{aligned}
\operatorname{chomp}_{1}\left(\tau, \mathrm{b}_{0}\right)= & \Sigma x:\left\{y: \epsilon|| y \cdot p k t_{i n} \mid+1=1\right\} \cdot\left\{z: \epsilon|| x \cdot p k t_{i n} \mid+1=1\right\}+ \\
& \Sigma x:\left\{y: \epsilon|| y \cdot p k t_{i n}|=1 \wedge| y \cdot p k t_{i n} \mid=0\right\} \cdot\left\{z: \epsilon|| x \cdot p k t_{i n} \mid=1\right\}
\end{aligned}
$$

Example. Given a type $\tau=\left\{x:\left\{y: \iota|| y . p k t_{\text {in }} \mid=8\right\} \mid x . p k t_{\text {in }}[0: 8]=x . \iota[4: 12]\right\}$

$$
\operatorname{chomp}_{1}\left(\tau, b_{0}\right)=\left\{x:\left\{y: \iota|| y \cdot p k t_{i n} \mid+1=8\right\} \mid b_{0}:: x \cdot p k t_{i n}[0: 7]=x . \iota[4: 12]\right\}
$$

Example. Given a header instance $A$ and a heap type $\tau=\left\{x: \epsilon \mid \mathrm{b}_{0}::\langle\rangle @ x . p k t_{\text {in }}[0]=10\right\}$. The first call to heapRef ${ }_{1}$ returns type $\left\{x: \epsilon \mid(y . l[0] @\langle\rangle) @ x \cdot p k t_{\text {in }}[0]=10\right\}$.

3.6.2 Correctness of Chomp. We prove that chomp is correct with respect to chomp $\Downarrow$. Specifically, Lemma 3.2 states that-given some heap $h \in \llbracket \tau \rrbracket_{\mathcal{E}}$-there exists a corresponding heap $h^{\prime}$ in the semantics of the chomped type that is equivalent to the heap obtained after applying chomp ${ }^{\Downarrow}$ to $h$. Since chomp adds a refinement on $x . l$, we evaluate the chomped type in an environment, where $x$ maps to the heap in which $\iota$ contains the first $\operatorname{sizeof}(\iota)$ bits from $h\left(p k t_{i n}\right)$. This reflects the intuition that chomp populates the header instance $\iota$ with the first sizeof $(\iota)$ bits from the input packet.

Lemma 3.2 (Semantic Сномp). If $x$ does not appear free in $\tau$, then for all heaps $h \in \llbracket \tau \rrbracket_{\mathcal{E}}$ where $\left|h\left(p k t_{\text {in }}\right)\right| \geq \operatorname{sizeof}(\iota)$, there exists $h^{\prime} \in \llbracket \operatorname{chomp}(\tau, l, x) \rrbracket_{\mathcal{E}^{\prime}}$ such that $h^{\prime}=\operatorname{chomp}^{\Downarrow}(h$, sizeof $(\iota))$ where $\mathcal{E}^{\prime}=\mathcal{E}\left[x \mapsto\left(\langle\rangle,\langle\rangle,\left[\iota \mapsto h\left(p k t_{\text {in }}\right)[0: \operatorname{sizeof}(\iota)]\right]\right)\right]$.

\subsection{Safety of $\Pi 4$}

We prove safety of $\Pi 4$ in terms of standard progress and preservation theorems. That is, well-typed programs do not get stuck and when well-typed programs are evaluated, they remain well typed. Both theorems make use of the entailment relation defined in Figure 9.

Theorem 3.3 (Progress). If $\cdot \vdash c:\left(x: \tau_{1}\right) \rightarrow \tau_{2}$ and $(I, O, H) \vDash \tau_{1}$, then either $c=$ skip or $\exists\left\langle I^{\prime}, O^{\prime}, H^{\prime}, c^{\prime}\right\rangle .\langle I, O, H, c\rangle \rightarrow\left\langle I^{\prime}, O^{\prime}, H^{\prime}, c^{\prime}\right\rangle$.

Proof. By induction on the typing derivation. For details, see the companion technical report. 
As usual, progress says that if a command is well-typed, it is either skip or can take a step.

Theorem 3.4 (Preservation). If $\Gamma \vdash c:\left(x: \tau_{1}\right) \rightarrow \tau_{2},\langle I, O, H, c\rangle \rightarrow\left\langle I^{\prime}, O^{\prime}, H^{\prime}, c^{\prime}\right\rangle$, and $\mathcal{E}=\Gamma$ and $(I, O, H) \models_{\mathcal{E}} \tau_{1}$, then there exists $\Gamma^{\prime}, \mathcal{E}^{\prime}, x^{\prime}, \tau_{1}^{\prime}, \tau_{2}^{\prime}$, such that $\Gamma^{\prime} \vdash c^{\prime}:\left(x^{\prime}: \tau_{1}^{\prime}\right) \rightarrow \tau_{2}^{\prime}$ and $\mathcal{E}^{\prime} \vDash \Gamma^{\prime}$ and $\left(I^{\prime}, O^{\prime}, H^{\prime}\right) \vDash_{\mathcal{E}^{\prime}} \tau_{1}^{\prime}$ and $\llbracket \tau_{2}^{\prime} \rrbracket_{\mathcal{E}^{\prime}\left[x^{\prime} \mapsto\left(I^{\prime}, O^{\prime}, H^{\prime}\right)\right]} \subseteq \llbracket \tau_{2} \rrbracket_{\mathcal{E}[x \mapsto(I, O, H)]}$

Proof. By induction on the typing derivation. For details, see the companion technical report.

The preservation theorem says that if a command is a well-typed command $c$ that can step to $c^{\prime}$, and a heap entails the input type $\tau_{1}$, then $c^{\prime}$ is well-typed from $\tau_{1}^{\prime}$ to $\tau_{2}^{\prime}$ for some $\tau_{1}^{\prime}$ and $\tau_{2}^{\prime}$ such that the final heap entails $\tau_{1}^{\prime}$ and the set of heaps described by $\tau_{2}^{\prime}$ is a subset of the heaps denoted by $\tau_{2}$ with their respective input heaps bound to variable $x$.

\section{IMPLEMENTATION}

We have built a prototype implementation of $\Pi 4$ 's type system in OCaml and Z3. Under the hood, it uses an encoding of $\Pi 4$ 's types into a decidable theory of first-order logic, facilitating use of an SMT solver to automatically discharge the various side conditions that arise during type checking. We describe the algorithmic type system and its decidability, some optimizations we use to simplify our types, and our $\mathrm{P}_{46}$ frontend.

\subsection{Algorithmic Type System and Decidability}

For our implementation, we define an algorithmic version of our type system whose rules are mostly identical to the rules from our declarative type system. Figure 10 shows two selected algorithmic typing rules that demonstrate the key differences from our declarative system. Many of the typing rules have semantic conditions that must be checked during type checking. In the algorithmic type system, we encode these constraints as subtype constraints. For example, when we type-check the command add $(\iota)$, we must check that the newly added instance is not already valid in the input type $\tau_{1}$, i.e., Excludes $\Gamma \tau_{1} \iota$. As shown by rule T-ADD-Algo in Figure 10, Excludes $\Gamma \tau_{1} \iota$ becomes the subtype check $\Gamma \vdash \tau_{1}<:\{x: \top \mid \neg x .$. valid $\}$. Similarly, rule T-Mod and T-Remit require Includes $\Gamma \tau_{1} l$, which becomes $\Gamma \vdash \tau_{1}<:\{x: \top \mid x$.l.valid $\}$ in T-Mod-Algo and TRemit-Algo respectively. The check sizeof $p k t_{i n}(\tau) \geq \operatorname{sizeof}(\iota)$ required by T-ExTRACT becomes $\Gamma \vdash \tau_{1}<:\left\{x: \top|| x . p k t_{i n} \mid \geq \operatorname{sizeof}(\iota)\right\}$ in rule T-Extract-Algo.

The second major difference is the rule for type ascription T-AsCRIBE-Algo. In our implementation we check if the input type $\tau_{1}^{\prime}$ is a subtype of the ascribed input type $\tau_{1}$. We then use the ascribed input type to compute an output type $\tau_{2}$. Finally, we check if the computed output type is a subtype of the ascribed output type. Note, that our type checking algorithm can be used to obtain a weak form of type inference. Given an input type that describes the state before the execution, our algorithm computes an output type, which describes the state after the execution of the program. However, a full-blown treatment of type reconstruction (such as the one used by Liquid Haskell [Vazou et al. 2018]) is left for future work.

We convert every check $\Gamma \vdash \tau_{1}<: \tau_{2}$ into a formula in the theory of fixed-width bit vectors. This is largely straightforward, except for the encoding of $p k t_{\text {in }}$ and $p k t_{\text {out }}$, which may be arbitrarily long. However, network switches have a maximum number of bits that they can receive or transmit, called the maximum transmission unit (MTU). So when compiling a P4 program to a given switch, we know that the transmitted packets must be smaller than MTU. We exploit this fact to prove a bound on the size of the bit vectors that must be considered.

More formally, we say that a type $\tau$ is bounded by $N$ in a context $\Gamma$, written $\Gamma \vdash \tau \leq N$, iff for every $\mathcal{E} \vDash \Gamma$, and $h \in \llbracket \tau \rrbracket_{\mathcal{E}},\left|h\left(p k t_{\text {in }}\right)\right|+\left|h\left(p k t_{\text {out }}\right)\right| \leq N$. We need to bound both $h\left(p k t_{\text {in }}\right)$ and $h\left(p k t_{\text {out }}\right)$ by $N$ because (as seen in the reset command), the emitted packet is $h\left(p k t_{\text {out }}\right) @ h\left(p k t_{\text {in }}\right)$. 
T-ADD-Algo

$\frac{\Gamma \vdash \tau_{1}<:\{x: \top \mid \neg x . \iota . v a l i d\} \quad \text { init }_{\mathcal{H} \mathcal{T}(\iota)}=v}{\Gamma \vdash \operatorname{add}(\iota):\left(x: \tau_{1}\right) \rightsquigarrow \Sigma y:\left\{z: \tau_{1} \mid z \equiv x\right\} .\left\{x: \iota \mid z \cdot p k t_{\text {in }}=z \cdot p k t_{\text {out }}=\langle\rangle \wedge z . \iota=v\right\}}$

T-Ascribe-Algo
$\frac{\Gamma \vdash c:\left(x: \tau_{1}\right) \rightsquigarrow \tau_{2}^{\prime} \quad \Gamma \vdash \tau_{1}^{\prime}<: \tau_{1} \quad \Gamma,\left(x: \tau_{1}^{\prime}\right) \vdash \tau_{2}^{\prime}<: \tau_{2}}{\Gamma \vdash c \text { as }\left(x: \tau_{1}\right) \rightarrow \tau_{2}:\left(x: \tau_{1}^{\prime}\right) \rightsquigarrow \tau_{2}}$

Fig. 10. Selected rules of the algorithmic type system

Theorem 4.1 (MTU-Bound) says that given an algorithmic typing judgement on a program $c$, for which the input type and all ascribed types in $c$ respect the MTU $N$, the output type will require no more than $N+\operatorname{emit}(c)$ bits, where emit $(c) \in \mathbb{N}$ is the number of bits that could possibly be emitted in $c$. The details are shown in the companion technical report. Note that even though the real input type is constrained by the same MTU $N$, intermediate states may require more than just $N$ bits. MTU-Bound shows that $N+\operatorname{emit}(c)$ suffices as the maximum combined width of $p k t_{\text {in }}$ and $p k t_{\text {out }}$.

Theorem 4.1 (MTU-Bound). For every $\Gamma, c, x, \tau_{1}, \tau_{2}$, and $N$, if $\Gamma \vdash \tau_{1} \leq N$ and $\Gamma \vdash c:\left(x: \tau_{1}\right) \rightsquigarrow \tau_{2}$ and every ascribed type in $c$ is also bounded by $N$, then $\Gamma,\left(x: \tau_{1}\right) \vdash \tau_{2} \leq N+\operatorname{emit}(c)$.

Proof. By induction on the typing derivation. For details, see the companion technical report.

Theorem 4.2 establishes the correctness of the algorithmic typing relation. It states that a program $c$ typechecks in the declarative system with type $\left(x: \tau_{1}\right) \rightarrow \tau_{2}$ if and only if it also typechecks in the algorithmic system with type $\left(x: \tau_{1}\right) \rightsquigarrow \tau_{2}^{\prime}$ and the output type of the algorithmic system $\tau_{2}^{\prime}$ is a subtype of the output type $\tau_{2}$ of the declarative system.

Theorem 4.2 (Algorithmic Typing Correctness). For all $\Gamma, c, x, \tau_{1}$, and $\tau_{2}$, where $x$ is not free in $\tau_{1}, \Gamma \vdash c:\left(x: \tau_{1}\right) \rightarrow \tau_{2}$ if and only if there is some $\tau_{2}^{\prime}$ such that $\Gamma \vdash c:\left(x: \tau_{1}\right) \rightsquigarrow \tau_{2}^{\prime}$, and $\Gamma,\left(x: \tau_{1}\right) \vdash \tau_{2}^{\prime}<: \tau_{2}$.

Proof. By induction on the typing derivation. For details, see the companion technical report.

With Theorems 4.2 and 4.1 in hand, it is straightforward to show the decidability of the declarative type system, i.e., that $\Gamma \vdash c:\left(x: \tau_{1}\right) \rightarrow \tau_{2}$ is decidable (cf. Theorem 4.3). Theorem 4.2 allows us to equivalently show that typechecking the command in the algorithmic type system-i.e., $\Gamma \vdash c:\left(x: \tau_{1}\right) \rightsquigarrow \tau_{2}^{\prime}$-terminates and that checking $\Gamma,\left(x: \tau_{1}\right) \vdash \tau_{2}^{\prime}<: \tau_{2}$ terminates, which follows by finite enumeration using the bounds guaranteed by Theorem 4.1 .

Theorem 4.3 (Decidability). If $\Gamma, \tau_{1}, \tau_{2}$ and every ascribed type in c are bounded by the MTU $N$, then $\Gamma \vdash c:\left(x: \tau_{1}\right) \rightarrow \tau_{2}$ is decidable.

Proof. Proof by Algorithmic Typing Correctness, MTU-Bound and by induction on the typing derivation. For details, see the companion technical report.

\subsection{Rewriting Optimizations}

The final major difference between the declarative type system and our implementation is that we exploit two type equivalences to eliminate $\Sigma$-types and chomp. We exploit the fact that $\Sigma$-types can be written using refinement and substitution types. In other words, in any context $\Gamma$, the type $\Sigma x: \tau_{1} \cdot \tau_{2}$ is equivalent to 


$$
\left\{x: \top \mid\left(\begin{array}{l}
x . p k t_{\text {in }}=l . p k t_{\text {in }} @ r . p k t_{\text {in }} \wedge \\
x . p k t_{\text {out }}=l . p k t_{\text {out }} @ r . p k t_{\text {out }}
\end{array}\right) \wedge \bigwedge_{\iota \in \operatorname{dom}(\mathcal{H} \mathcal{T})}\left(\begin{array}{l}
\text { x.l.valid }=\text { l.l.valid } \oplus \text { r.l.valid } \wedge \\
\text { l.l.valid } \Longrightarrow x . \iota=l . \iota \wedge \\
r . \text {.l.valid } \Longrightarrow x . l=r . \iota
\end{array}\right)\right\} \begin{aligned}
& \left.r \mapsto \tau_{2}\right] \\
& {\left[l \mapsto \tau_{1}\right]}
\end{aligned}
$$

We also can eliminate occurrences of chomp produced by extractions. Observe that in the context where $(x: \tau)$, the following two types are equivalent

$$
\begin{gathered}
\left.\sum y:\left\{\begin{array}{l|l}
z: \iota \mid & \begin{array}{c}
z \cdot p k t_{\text {in }}=\langle\rangle \wedge \\
z \cdot p k t_{\text {out }}=\langle\rangle
\end{array}
\end{array}\right\} \cdot\left\{\begin{array}{c}
z: \operatorname{chomp}(\tau, \iota, y) \mid\left(\begin{array}{c}
y \cdot \iota @ z \cdot p k t_{\text {in }}=x \cdot p k t_{\text {in }} \wedge \\
z \cdot p k t_{\text {out }}=x \cdot p k t_{\text {out }}
\end{array}\right.
\end{array}\right) \wedge z \equiv_{\iota} x\right\} \\
\doteq
\end{gathered}
$$

Because these types are equivalent we can give extract $(l)$ commands the following type:

$$
(x: \tau) \rightarrow\left\{y: \top \mid y . \iota . v a l i d \wedge x . p k t_{\text {in }}=y . \iota @ y . p k t_{\text {in }} \wedge x \cdot p k t_{\text {out }}=y \cdot p k t_{\text {out }} \wedge \bigwedge_{\kappa \in \operatorname{dom}(\mathcal{H} \mathcal{T}) \wedge \kappa \neq \iota} y \cdot \kappa=x . \kappa\right\}
$$

This optimization, along with similar changes to the types for $\operatorname{add}(\iota)$ and remit $(\iota)$ greatly reduce the size of the generated $\mathrm{Z} 3$ formulae, making typechecking tractable.

\subsection{Beyond the Core Calculus}

Our prototype is equipped with a $\mathrm{P} 4_{16}$ frontend that uses Petr4's parser [Doenges et al. 2021] to translate a subset of type-annotated $\mathrm{P} 4_{16}$ programs into $\Pi 4$ programs. We leverage $\mathrm{P} 4$ 's builtin annotation mechanism to allow users to annotate control and parser blocks with types using the custom @pi4( $\sigma)$ annotation, where $\sigma$ is the desired type. We also provide convenience notation such as @pi4_roundtrip $(\tau)$, which ensures, as elaborated in Section 5.3 that the composition of deparser, reset, and parser has type $(x: \tau) \rightarrow\{y: \top \mid x \equiv y\}$.

\section{CHECKING NETWORK INVARIANTS}

We now show that dependent types are a good match for P4, by demonstrating that $\Pi 4$ 's type system can be used to (i) check real network protocol invariants and (ii) verify a variety of basic and advanced safety properties. We showcase properties that are also studied in the context of other P4 verification tools [Liu et al. 2018; Stoenescu et al. 2018]. All examples, in this section and the next, have been implemented in our $\Pi 4$ prototype.

In most $\mathrm{P} 4$ programs, packet-forwarding behavior is specified using a predefined record of type standard_metadata_t. In particular, the egress_spec field instructs the switch to forward the packet out on a specific port. We assume that the field is initialized to $0 \times 00$, indicating that no forwarding decision has been made, and that by setting the field to $0 \times 1 \mathrm{FF}$ (i.e., the largest unsigned 9-bit value), the switch can be instructed to drop the packet. ${ }^{4}$ For simplicity, we treat all P4 metadata as an ordinary header instance called stdmeta.

\subsection{Protocol Conformance}

We start with examples showing how П4's type system can be used to ensure that a program conforms with standard network protocols.

\footnotetext{
${ }^{4}$ In the examples that follow, we use bitvector literals assuming that they are implicitly cast to the appropriate widths (following P4 16 's casting semantics). The implementation, however, requires these to be explicit lengths, e.g. 0 b111111111 instead of $0 \times 1 \mathrm{FF}$ for a 9 bit field.
} 

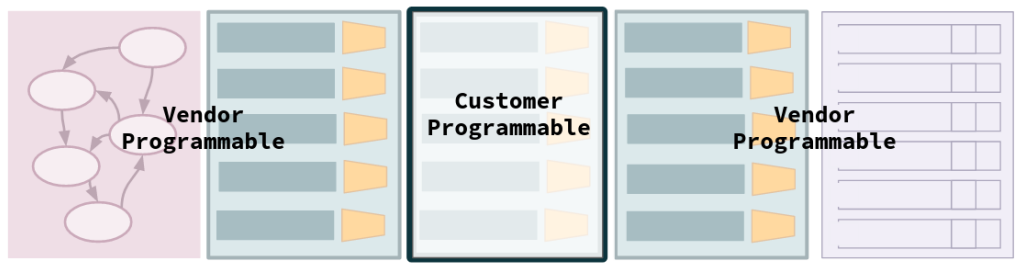

Fig. 17. Modular router design

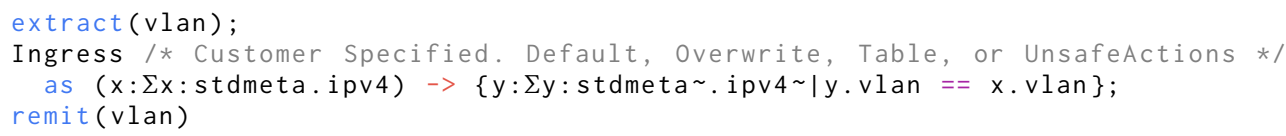

Fig. 18. Instantiation of modular router design; the parser (Pre) and deparser (Post) are provided by the vendor, the Ingress code is provided by the customer.

function interface into the hardware), and registers. The unpredictability of hash functions is difficult to verify, but we can either over-approximate them as uninterpreted functions, or use a more fine-grained approach such as concolic verification. Externs either need to be annotated with specific types, or over-approximated as uninterpreted functions. Registers are on-switch state that can be modified by the packet or the controller and persists between packets. This is tricky to represent in the semantics and has some distributed computing concerns. We could over-approximate the behavior by havoc-ing the values every time the register is read.

\section{MODULARITY REASONING WITH П4}

An emerging design pattern for data plane switches is partial programmability, e.g., Cisco's daPIPE [Baldi 2019], which is designed for the Nexus 3400 switch [Cisco 2018]. The idea is that a device vendor provides a partially-implemented pipeline together with a set of program points where customers can inject custom code as shown in Figure 17. The designer requires that customer programs satisfy certain properties, but in current architectures, they are not automatically checked.

To illustrate, consider a deployment of the customizable pipeline in a campus network where network engineers want to experiment with in-band network telemetry (INT) without perturbing the VLAN tag, which is used to enforce security policies. Let's say there are four classes of traffic, Visitor, Student, Faculty, and Staff, each with unique VLAN identifiers. We want to ensure that no matter how Ingress is instantiated in the left of Figure 18, it cannot cause students and visitors to acquire privileges of faculty or staff-e.g., this might leak confidential data. With $\Pi 4$, we can design a modular system that statically checks invariants on customer programs. Practically, we can ensure that VLAN is not changed by checking that customer's code has a type like: $(x: \tau) \rightarrow\left\{y: \tau^{\prime} \mid x . v l a n . v i d=y . v l a n . v i d\right\}$, where the $\tau$ and $\tau^{\prime}$ are appropriate for the specific pipeline. We check, once-and-for-all, that the surrounding switch code composes with this type, and incrementally check that the customer code has this type (for an appropriate $\tau$ ).

\subsection{Specifying an Invariant}

To further illustrate, consider the toy example shown the right of Figure 18, which has a VLAN instance ( 32 bits) and the standard metadata used in the P4 switch model (325 bits), including a 9-bit egress specification stdmeta.egress_spec, and a 12-bit vlan tag field, vlan.vid. The control flow simply extracts the VLAN instance, executes the modular Ingress control, and then emits 
we create a new header_vlan_table with a 12-bit field vid_key and a 1-bit field act, modelling the table application via the code shown in the bottom left of Figure 19. This will typecheck since no branch of the code modifies the vlan.vid field, and _vlan_table is permitted to be valid.

Unsafe Actions. Now consider a vlan table where each action does modify the VLAN id. For example, the table shown in the bottom right of Figure 18 can either set the VID to one of \{Faculty, Staff\}, or, if the packet misses in the table, to Visitor. Here, the VLAN id is clobbered whenever this table is applied, triggering a violation of the subset check just as with Overwrite.

\section{RELATED WORK}

Formal Reasoning for P4 Programs. A number of verification approaches have been proposed for P4 programs. p4v [Liu et al. 2018] applies classical techniques based on predicate transformer semantics to achieve monolithic verification of P4 programs. Vera [Stoenescu et al. 2018] and P4-Assert [Freire et al. 2018] are symbolic execution engines for P4. The bf 4 tool [Dumitrescu et al. 2020] follows the approach of $\mathrm{p} 4 \mathrm{v}$, but also attempts to infer control-plane constraints that are sufficiently strong to establish correctness, and offers heuristics for repairing programs when verification fails. SafeP4 [Eichholz et al. 2019] uses a simple type system to track header validity. Petr4 [Doenges et al. 2021] develops a formal semantics for P4 but does not itself offer verification tools. In constrast to this earlier work, $\Pi 4$ uses dependent types and offers compositional verification.

Dependent Types. Early work by Xi and Pfenning [1999] showed how dependent types could be used to eliminate run-time safety checks-e.g., array bounds checks in imperative programs. Xanadu [Xi 2000] adds dependent typing to imperative programming, but does not capture the effect of mutations in the type. Xi and Harper [2001] later showed how dependent types could be applied to assembly code. Deputy [Condit et al. 2007] used dependent types to reason about complex, heap-allocated data structures. Similar to Deputy, $\Pi 4$ 's typing rule for modification of header fields is also inspired by the Hoare axiom for assignment. $\Pi 4$ is different in that typechecking has no effect on the run time and also supports path-sensitive reasoning. Similar to П4, Hoare Type Theory (HTT) [Nanevski et al. 2006] statically tracks how the heap evolves during execution. Typing of computations in HTT is similar to the dependent function types $\Pi 4$ - the type captures the state before and after execution, possibly relating the output type with the input type. In our domain, this requires bit-by-bit transformations on the input type, provided by chomp. Other type systems like Ynot [Nanevski et al. 2008], FCSL [Nanevski et al. 2014], and F* [Swamy et al. 2016] provide dependent types for low-level imperative programming. They target general functional verification and often require manual programs-as-proofs to do so. $\Pi 4$ is designed with domainspecific properties of network programming in mind and is fully automatic.

Solver-Aided Tools. Recent work on dependently-typed languages has focused on automation, building on advances in SAT/SMT solvers to make dependent types usable by ordinary programs. A prominent example is Liquid Haskell [Rondon et al. 2008], which extends Haskell with decidable refinement types. Under the hood, proof obligations generated during type checking are transparently handled by an SMT solver. Just as Liquid Haskell requires its refinements to be in the theory of quantifier-free integer linear arithmetic in order to be decidable, $\Pi 4$ stipulates that types must denote finite sets-a restriction justified by the domain. This assumption lets us encode types into the effectively propositional fragment of first-order logic over bit vectors.

Formalizing Protocols. Another line of work focuses on language-based specifications of protocols. CMU's FoxNet project used SML to specify the behavior of an entire networking stack [Biagioni et al. 1994]. McCann and Chandra [2000] used a type-based approach to give abstract specifications of protocols. Grammar-based tools such as PADS [Fisher and Gruber 2005], Narcissus [Delaware 
et al. 2019], and Yakker [Jim et al. 2010], enable specifying the syntax of complex, dependent formats including network protocols, and provide tools for serializing and deserializing data. They focus exclusively on deriving correct parsers from a typed representation of data and may be suitable to describe the header formats and the parser, but there is no equivalent to our chomp operator that allows us to statically capture how the input packet changes during parsing.

\section{CONCLUSION AND FUTURE WORK}

This paper presented $\Pi 4$-the first dependently-typed language for data plane programming-a domain with difficult challenges where programming language theory can have a big impact. In particular, low-level data plane languages like P4 seem to be a sweet spot for dependent types. On the one hand, precise types are necessary because critical correctness properties often hinge on intricate, bit-level packet formats, where the first few bits of a packet determine the format, the length, and the processing of the following ones. On the other hand, a high degree of automation is possible due to the restricted nature of the language, which does not support pointers, loops, or other features that often complicate very precise type systems. Yet, thus far, dependent typing has not been explored for data plane programs-the community has relied on verification tools that lack compositional reasoning.

П4's type system is innovative in its combination of refinement types, dependent function types, a limited form of regular types, including unions, explicit substitutions, and a primitive "chomp" operation, reminiscent of regular expression derivatives [Brzozowski 1964], which can be used to give a precise type to P4's parsing constructs. It is capable of statically checking advanced properties of data plane programs that combine packet serialization and deserialization operations with imperative control-flow. Under the hood, an SMT solver automatically discharges the formulas generated during type checking without requiring any manual proof. We define $\Pi 4$ formally and prove type soundness and decidability. Our case studies demonstrate how $\Pi 4$ supports modular reasoning in scenarios ranging from basic safety properties to intricate invariants.

There are a number of interesting directions for future work. We plan to investigate connections that our verified approach to parsing using derivatives may have to other domains, e.g., verified serializers and deserializers like EverParse [Ramananandro et al. 2019] and Narcissus [Delaware et al. 2019]. Another direction is to consider the effect of modularity on verification times; if a tool incrementally caches verification results for ascribed code blocks, it would only have to check the portions of the code that change between runs of the typechecker. Some preliminary experiments indicate that modular typechecking offers significant benefits, but an empirical study to answer this question carefully is left for future work, after we have optimized our prototype. We also plan to extend $\Pi 4$ to handle more complicated features of $\mathrm{P} 4$ perhaps requiring concolic techniques [Godefroid et al. 2005]. Further, understanding whether dependent types are the right interface for modular verification of dataplane programs is important. In fact, user studies investigating the appropriate typing interfaces, such as gradual typing and type inference, would be important for guiding the design of impactful systems for modular data plane verification.

\section{ACKNOWLEDGMENTS}

We are grateful to the POPL reviewers for their careful feedback and many suggestions for improving this paper. Our work has been supported in part by the German Research Foundation (DFG) as part of the Collaborative Research Center (CRC) 1053 MAKI, by the National Research Center for Applied Cybersecurity ATHENE, by the National Science Foundation under grant FMiTF-1918396 as well as a Graduate Research Fellowship, the Defense Advanced Research Projects Agency under Contract HR001120C0107, and gifts from Keysight and InfoSys. 


\section{REFERENCES}

M. Baldi. 2019. daPIPE a Data Plane Incremental Programming Environment. In Symposium on Architectures for Networking and Communications Systems (ANCS). 1-6. https://doi.org/10.1109/ANCS.2019.8901893

Ryan Beckett and Ratul Mahajan. 2020. A General Framework for Compositional Network Modeling. In Workshop on Hot Topics in Networks (HotNets). 8-15. https://doi.org/10.1145/3422604.3425930

Edoardo Biagioni, Robert Harper, Peter Lee, and Brian G. Milnes. 1994. Signatures for a Network Protocol Stack: A Systems Application of Standard ML. In Conference on LISP and Functional Programming (LFP). 55-64. https://doi.org/10.1145/ 182409.182431

Pat Bosshart, Dan Daly, Glen Gibb, Martin Izzard, Nick McKeown, Jennifer Rexford, Cole Schlesinger, Dan Talayco, Amin Vahdat, George Varghese, and David Walker. 2014. P4: Programming Protocol-Independent Packet Processors. SIGCOMM Computer Communications Review (CCR) (July 2014), 87-95. https://doi.org/10.1145/2656877.2656890

Pat Bosshart, Glen Gibb, Hun-Seok Kim, George Varghese, Nick McKeown, Martin Izzard, Fernando Mujica, and Mark Horowitz. 2013. Forwarding Metamorphosis: Fast Programmable Match-Action Processing in Hardware for SDN. In Conference of the Special Interest Group on Data Communication on the Applications, Technologies, Architectures, and Protocols for Computer Communication (SIGCOMM). 99-110. https://doi.org/10.1145/2486001.2486011

Janusz A. Brzozowski. 1964. Derivatives of Regular Expressions. F. ACM 11, 4 (Oct. 1964), 481-494. https://doi.org/10.1145/ 321239.321249

Giuseppe Castagna, Kim Nguyen, Zhiwu Xu, Hyeonseung Im, Sergueï Lenglet, and Luca Padovani. 2014. Polymorphic Functions with Set-Theoretic Types: Part 1: Syntax, Semantics, and Evaluation. In Symposium on Principles of Programming Languages (POPL). 5-17. https://doi.org/10.1145/2535838.2535840

Cisco. 2018. Cisco Nexus 3000 Series Switches. https://www.cisco.com/c/en/us/products/switches/nexus-3000-seriesswitches/index.html

Jeremy Condit, Matthew Harren, Zachary Anderson, David Gay, and George C. Necula. 2007. Dependent Types for Low-Level Programming. In European Symposium on Programming (ESOP). 520-535. https://doi.org/10.1007/978-3-540-71316-6_35

Benjamin Delaware, Sorawit Suriyakarn, Clément Pit-Claudel, Qianchuan Ye, and Adam Chlipala. 2019. Narcissus: Correctby-Construction Derivation of Decoders and Encoders from Binary Formats. Proceedings of the ACM Programming Languages (PACMPL) 3, ICFP, Article 82 (July 2019). https://doi.org/10.1145/3341686

Ryan Doenges, Mina Tahmasbi Arashloo, Santiago Bautista, Alexander Chang, Newton Ni, Samwise Parkinson, Rudy Peterson, Alaia Solko-Breslin, Amanda Xu, and Nate Foster. 2021. Petr4: Formal Foundations for P4 Data Planes. Proceedings of the ACM on Programming Languages (PACMPL) 5, POPL, Article 41 (Jan. 2021). https://doi.org/10.1145/ 3434322

Dragos Dumitrescu, Radu Stoenescu, Lorina Negreanu, and Costin Raiciu. 2020. Bf4: Towards Bug-Free P4 Programs. In Conference of the Special Interest Group on Data Communication on the Applications, Technologies, Architectures, and Protocols for Computer Communication (SIGCOMM). 571-585. https://doi.org/10.1145/3387514.3405888

Matthias Eichholz, Eric Campbell, Nate Foster, Guido Salvaneschi, and Mira Mezini. 2019. How to Avoid Making a BillionDollar Mistake: Type-Safe Data Plane Programming with SafeP4. In European Conference on Object-Oriented Programming (ECOOP). 12:1-12:28. https://doi.org/10.4230/LIPIcs.ECOOP.2019.12

Robert Ennals, Richard Sharp, and Alan Mycroft. 2004. Linear Types for Packet Processing. In European Symposium on Programming (ESOP). 204-218. https://doi.org/10.1007/978-3-540-24725-8_15

Kathleen Fisher and Robert Gruber. 2005. PADS: A Domain-Specific Language for Processing Ad Hoc Data. In Conference on Programming Language Design and Implementation (PLDI). 295-304. https://doi.org/10.1145/1065010.1065046

Lucas Freire, Miguel Neves, Lucas Leal, Kirill Levchenko, Alberto Schaeffer-Filho, and Marinho Barcellos. 2018. Uncovering Bugs in P4 Programs with Assertion-Based Verification. In Symposium on SDN Research (SOSR). Article 4, 7 pages. https://doi.org/10.1145/3185467.3185499

Jiaqi Gao, Ennan Zhai, Hongqiang Harry Liu, Rui Miao, Yu Zhou, Bingchuan Tian, Chen Sun, Dennis Cai, Ming Zhang, and Minlan Yu. 2020. Lyra: A Cross-Platform Language and Compiler for Data Plane Programming on Heterogeneous ASICs. In Conference of the Special Interest Group on Data Communication on the Applications, Technologies, Architectures, and Protocols for Computer Communication (SIGCOMM). 435-450. https://doi.org/10.1145/3387514.3405879

Vladimir Gapeyev and Benjamin C. Pierce. 2003. Regular Object Types. In European Conference on Object-Oriented Programming (ECOOP). 151-175. https://doi.org/10.1007/978-3-540-45070-2_8

Patrice Godefroid, Nils Klarlund, and Koushik Sen. 2005. DART: Directed automated random testing. In Conference on Programming Language Design and Implementation (PLDI). 213-223. https://doi.org/10.1145/1065010.1065036

Toke Høiland-Jørgensen, Jesper Dangaard Brouer, Daniel Borkmann, John Fastabend, Tom Herbert, David Ahern, and David Miller. 2018. The EXpress Data Path: Fast Programmable Packet Processing in the Operating System Kernel. In International Conference on Emerging Networking EXperiments and Technologies (CoNEXT). 54-66. https://doi.org/10. 1145/3281411.3281443 
Haruo Hosoya and Benjamin C. Pierce. 2003. XDuce: A Statically Typed XML Processing Language. ACM Transactions on Internet Technology (TOIT) 3, 2 (May 2003), 117-148. https://doi.org/10.1145/767193.767195

Stephen Ibanez, Gordon Brebner, Nick McKeown, and Noa Zilberman. 2019. The P4->NetFPGA Workflow for Line-Rate Packet Processing. In Symposium on Field-Programmable Gate Arrays (FPGA). 1-9. https://doi.org/10.1145/3289602.3293924

Trevor Jim, Yitzhak Mandelbaum, and David Walker. 2010. Semantics and Algorithms for Data-Dependent Grammars. In Symposium on Principles of Programming Languages (POPL). 417-430. https://doi.org/10.1145/1706299.1706347

Xin Jin, Xiaozhou Li, Haoyu Zhang, Robert Soulé, Jeongkeun Lee, Nate Foster, Changhoon Kim, and Ion Stoica. 2017. NetCache: Balancing Key-Value Stores with Fast In-Network Caching. In Symposium on Operating Systems Principles (SOSP). 121-136. https://doi.org/10.1145/3132747.3132764

Jed Liu, William Hallahan, Cole Schlesinger, Milad Sharif, Jeongkeun Lee, Robert Soulé, Han Wang, Călin Caşcaval, Nick McKeown, and Nate Foster. 2018. p4v: Practical Verification for Programmable Data Planes. In Conference of the Special Interest Group on Data Communication on the Applications, Technologies, Architectures, and Protocols for Computer Communication (SIGCOMM). 490-503. https://doi.org/10.1145/3230543.3230582

Conor McBride. 2001. The derivative of a regular type is its type of one-hole contexts. Extended abstract, available at http://strictlypositive.org/diff.pdf.

Peter J. McCann and Satish Chandra. 2000. Packet Types: Abstract Specification of Network Protocol Messages. In Conference of the Special Interest Group on Data Communication on the Applications, Technologies, Architectures, and Protocols for Computer Communication (SIGCOMM). 321-333. https://doi.org/10.1145/347059.347563

Chitra Muthukrishnan, Vern Paxson, Mark Allman, and Aditya Akella. 2010. Using Strongly Typed Networking to Architect for Tussle. In Workshop on Hot Topics in Networks (HotNets). Article 9, 6 pages. https://doi.org/10.1145/1868447.1868456

Aleksandar Nanevski, Ruy Ley-Wild, Ilya Sergey, and Germán Andrés Delbianco. 2014. Communicating State Transition Systems for Fine-Grained Concurrent Resources. In European Symposium on Programming (ESOP). 290-310. https: //doi.org/10.1007/978-3-642-54833-8_16

Aleksandar Nanevski, Greg Morrisett, and Lars Birkedal. 2006. Polymorphism and Separation in Hoare Type Theory. In International Conference on Functional Programming (ICFP). 62-73. https://doi.org/10.1145/1159803.1159812

Aleksandar Nanevski, Greg Morrisett, Avraham Shinnar, Paul Govereau, and Lars Birkedal. 2008. Ynot: Dependent Types for Imperative Programs. In International Conference on Functional Programming (ICFP. 229-240. https://doi.org/10.1145/ 1411204.1411237

Tahina Ramananandro, Antoine Delignat-Lavaud, Cedric Fournet, Nikhil Swamy, Tej Chajed, Nadim Kobeissi, and Jonathan Protzenko. 2019. EverParse: Verified Secure Zero-Copy Parsers for Authenticated Message Formats. In USENIX Security Symposium (USENIX Security). 1465-1482. https://www.usenix.org/conference/usenixsecurity19/presentation/delignatlavaud

Patrick M. Rondon, Ming Kawaguci, and Ranjit Jhala. 2008. Liquid Types. In Conference on Programming Language Design and Implementation (PLDI). 159-169. https://doi.org/10.1145/1375581.1375602

Hardik Soni, Myriana Rifai, Praveen Kumar, Ryan Doenges, and Nate Foster. 2020. Composing Dataplane Programs with $\mu \mathrm{P} 4$. In Conference of the Special Interest Group on Data Communication on the Applications, Technologies, Architectures, and Protocols for Computer Communication (SIGCOMM). 329-343. https://doi.org/10.1145/3387514.3405872

Radu Stoenescu, Dragos Dumitrescu, Matei Popovici, Lorina Negreanu, and Costin Raiciu. 2018. Debugging P4 Programs with Vera. In Conference of the Special Interest Group on Data Communication on the Applications, Technologies, Architectures, and Protocols for Computer Communication (SIGCOMM). 518-532. https://doi.org/10.1145/3230543.3230548

Nikhil Swamy, Cătălin Hrițcu, Chantal Keller, Aseem Rastogi, Antoine Delignat-Lavaud, Simon Forest, Karthikeyan Bhargavan, Cédric Fournet, Pierre-Yves Strub, Markulf Kohlweiss, Jean-Karim Zinzindohoue, and Santiago Zanella-Béguelin. 2016. Dependent Types and Multi-Monadic Effects in $\mathrm{F}^{*}$. In Symposium on Principles of Programming Languages (POPL). 256-270. https://doi.org/10.1145/2837614.2837655

Sam Tobin-Hochstadt and Matthias Felleisen. 2010. Logical Types for Untyped Languages. In International Conference on Functional Programming (ICFP). 117-128. https://doi.org/10.1145/1863543.1863561

Niki Vazou, Eric L. Seidel, Ranjit Jhala, Dimitrios Vytiniotis, and Simon Peyton-Jones. 2014. Refinement Types for Haskell. In International Conference on Functional Programming (ICFP). 269-282. https://doi.org/10.1145/2628136.2628161

Niki Vazou, Éric Tanter, and David Van Horn. 2018. Gradual Liquid Type Inference. Proceedings of the ACM on Programming Languages (PACMPL) 2, OOPSLA, Article 132 (Oct. 2018), 25 pages. https://doi.org/10.1145/3276502

Han Wang, Robert Soulé, Huynh Tu Dang, Ki-Suh Lee, Vishal Shrivastav, Nate Foster, and Hakim Weatherspoon. 2017. P4FPGA: A Rapid Prototyping Framework for P4. In Symposium on SDN Research (SOSR). 122-135. https://doi.org/10. $1145 / 3050220.3050234$

Hongwei Xi. 2000. Imperative programming with dependent types. In Symposium on Logic in Computer Science (LICS) 375-387. https://doi.org/10.1109/LICS.2000.855785

Hongwei Xi and Robert Harper. 2001. A Dependently Typed Assembly Language. In International Conference on Functional Programming (ICFP). 169-180. https://doi.org/10.1145/507635.507657 
Hongwei Xi and Frank Pfenning. 1999. Dependent Types in Practical Programming. In Symposium on Principles of Programming Languages (POPL). 214-227. 